\title{
Soporte ventilatorio en pacientes críticos con COVID-19: a 18 meses de la pandemia en Chile
}

\section{Respiratory support of the COVID-19 critically ill patient: 18 months since the pandemic started in Chile}

\author{
Rodrigo Ulloa MD. ${ }^{1}$, Pablo Born MD. ${ }^{1}$, Eduardo Kattan MDm, MMEd. ${ }^{1}$, Guillermo Bugedo MD. ${ }^{1, *}$ \\ 1 Departamento de Medicina Intensiva, Facultad de Medicina, Pontificia Universidad Católica de Chile.
}

Conflictos de interés: Los autores no presentan conflicto de interés.

Fecha de recepción: 16 de septiembre de 2021 / Fecha de aceptación: 16 de septiembre de 2021

\begin{abstract}
After 18 months from the beginning of the pandemic, we have increased our knowledge about different aspects of respiratory care of critically ill patients with SARS-CoV-2 pneumonia. In this review, we analyze the available evidence regarding physiopathology and clinical course of COVID-19 pneumonia; the pillars of respiratory support (both non-invasive and invasive); pharmacological therapy, and the challenges derived from the chronic critically ill COVID-19 patient. Even though there are still many questions left to be answered, as long as we keep learning from this new disease and its' impact on our organism, we will opportunely adapt our care strategies.
\end{abstract}

Key words: SARS-CoV-2, intensive care, mechanical ventilation; ARDS.

\section{RESUMEN}

Después de 18 meses de pandemia, hemos aprendido mucho sobre las distintas aristas del manejo ventilatorio del paciente crítico con neumonía por SARS-CoV-2. En esta revisión, analizamos la evidencia disponible en cuanto a la fisiopatología y curso clínico de la neumonía por COVID-19; los pilares del soporte ventilatorio no invasivo e invasivo; la terapia farmacológica; y los desafíos del paciente crítico crónico con COVID-19. Si bien aún quedan muchas preguntas por responder, a medida que sigamos aprendiendo sobre esta nueva enfermedad y su impacto en el organismo, iremos adaptando oportunamente nuestras estrategias de cuidado.

Palabras clave: SARS-CoV-2, cuidado intensivo, ventilación mecánica, SDRA.

\section{Introducción}

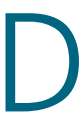

esde su aparición a fines de 2019 en Wuhan y en el verano de 2020 en Chile, la infección por el nuevo virus SARS-CoV-2 se ha llevado la vida de millones de personas a nivel mundial. Las unidades de cuidados intensivos y de urgencias de nuestro país, y toda la red hospitalaria fue estresada al máximo, y en muchos otros aspectos cambió la forma de vida de nuestra sociedad.

Sabiendo que es probable que enfrentemos nuevas olas este o los próximos años, comentaremos algunos aspectos clínicos de la neumonía asociada al virus SARS-CoV-2 (COVID-19) y sus procesos fisiopatológicos, así como sugerencias para enfrentar con éxito un paciente con insuficiencia respiratoria y aumento del trabajo ventilatorio, y requieren de soporte ventilatorio invasivo o no invasivo.

\section{La evolución del COVID-19 y sus fases}

Sin duda, el enfrentamiento actual a esta enfermedad es distinto al de los primeros meses porque hemos aprendido su comportamiento, desde su fisiopatología, las medidas preven- 
tivas hasta sus complicaciones. Sabemos que la insuficiencia respiratoria asociada a COVID-19 es una enfermedad que puede ser modulada con el uso de esteroides, que tiene un alto potencial trombótico, una alta prevalencia de delirium y complicaciones infecciosas, y requiriendo un alto porcentaje -como no habíamos visto en el pasado - de traqueostomía y estadías prolongadas en la unidad de cuidado intensivo[1],[2].

El COVID-19 severo involucra mayoritariamente manifestaciones respiratorias, aunque también se ven afectados otros sistemas, y la enfermedad aguda a menudo va seguida de complicaciones prolongadas. Las manifestaciones complejas sugieren que el SARS-CoV-2 desregula la respuesta del huésped, desencadenando trastornos inmuno-inflamatorios, trombóticos y parenquimatosos de amplio alcance[3],[4].

En los pacientes que desarrollan una neumonía, los síntomas respiratorios comienzan entre cinco y diez días después del contagio, detectándose tenues infiltrados en la radiografía de tórax. La tomografía computarizada nos refleja con mayor precisión el grado de compromiso pulmonar, así como el estadio, la presencia de enfermedad tromboembólica y otras complicaciones. En la fase aguda de la enfermedad predomina la exudación, con opacidades multifocales (densidad en "vidrio esmerilado") de predominio en la periferia pulmonar y áreas condensantes (Figuras 1 y 2). Hacia la segunda y tercera semana, aparecen condensación peribroncovascular y subpleural, y luego empedrado (crazy paving) y bronquiolectasias por tracción, determinadas por un proceso de neumonía en organización. Una tomografía computarizada (TC) en este período es fundamental para determinar el grado de organización y consecuentemente decidir aportes adicionales de esteroides (Figura 2). Sin embargo, la radiación ionizante es un tema que nos obliga a ser juiciosos y prudentes en su indicación.

La evolución posterior dependerá del curso de la enfermedad, el manejo ventilatorio, hemodinámico y nutricional, el uso de esteroides y la presencia de infecciones y otras complicaciones, y su manejo.

\section{La fase aguda y el soporte no invasivo}

La posición pronoasociada a oxigenoterapia, idealmente apoyada con cánula nasal de alto flujo (CNAF) puede prevenir la conexión al ventilador. Si bien esto es difícil de demostrar en un estudio prospectivo y aleatorizado, ya existe evidencia de buena calidad metodológica de su beneficio[5], y la amplia aplicación de esta técnica a nivel nacional es probablemente una de las razones que evitó que nuestro sistema de salud colapsara en el periodo más crítico de la pandemia[6].

El principal mecanismo de acción de la CNAF es la disminución del espacio muerto, lo que permite optimizar la ventilación alveolar y disminuir el trabajo ventilatorio[7]. Sumado a la posición prono, que optimiza la relación ventilación perfusión[8], es la terapia de primera línea para prevenir la caída al ventilador en pacientes con COVID-19, y posiblemente también para neumonías de otras causas.

Algunos detractores de esta técnica sugieren que retrasaría la conexión al ventilador, favoreciendo el daño pulmonar autoinducido por el paciente o P-SILI[9], y pudiera llevar eventualmente a ventilar pacientes con pulmones más dañados, empeorando su pronóstico. Nuestra hipótesis es que la neumonía asociada al SARS-CoV-2, al no tener una terapia específica, sigue su curso -a veces muy lento- independiente de la terapia ventilatoria (Figura 1)

La ventilación mecánica no invasiva (VNI), por otra parte, no cuenta con evidencia consolidada como una estrategia para enfrentar la insuficiencia respiratoria aguda hipoxémica y podría exponer al paciente a sostener volúmenes corrientes peligrosamente altos por largo tiempo[10],[11]. Sin embargo, evidencia reciente demuestra que la VNI vía Helmet puede ser beneficioso y comparable a CNAF en pacientes con falla respiratoria secundaria a COVID-19[12],[13].

\section{Indicación de IOT}

La indicación de intubación orotraqueal (IOT) fue tema de controversia al comienzo de esta pandemia. Mientras algunos sugerían intubar precozmente para proteger el pulmón y disminuir la dispersión de aerosoles, otros sugerían mantener los criterios clásicos de aumento del trabajo respiratorio, utilizando el posicionamiento en prono y métodos no invasivos para sostener la oxigenación[14],[15].

La indicación para determinar la IOT y conexión al ventilador son clínicas (Tabla 1). Criterios aislados en base a la oxigenación o la taquipnea, sin acompañarse de signos de mala tolerancia al aumento del trabajo ventilatorio, podrían llevar a intubaciones innecesarias. Sin embargo, la preservación de la ventilación espontánea en un paciente con gran demanda ventilatoria pasa a representar más un daño en comparación con la ventilación controlada del paciente sedado está lejos de estar bien definido[16].

\section{Ventilación mecánica}

\section{Daño inducido por la ventilación mecánica y ventilación protectora}

La ventilación mecánica con presión positiva es el pilar del manejo de la insuficiencia respiratoria COVID-19. Sin embargo, una mala programación del ventilador puede llevar a una elongación excesiva y patológica del tejido pulmonar, generando un daño mecánico con liberación de mediadores pro inflamatorios hacia la circulación sistémica, en lo que se ha llamado daño inducido por la ventilación mecánica (VILI)[17]. De aquí nace el concepto de ventilación protectora, que consiste en disminuir los niveles de volumen/presión aplicados sobre el tejido pulmonar del paciente, y cuyo pilar es la limitación del volumen corriente $(\mathrm{Vt})$, donde el uso de $6 \mathrm{ml} / \mathrm{kg}$ de peso ideal (IBW, de ideal body weight) mostró disminuir la mortalidad en comparación con $12 \mathrm{ml} / \mathrm{kg}$ IBW[18]. La limitación del Vt ha mostrado disminuir los todos los mecanismos fisiológicos de VILI[19]. La limitación de la driving pressure (DP, o presión meseta - PEEP) también ha mostrado ser un indicador de sobrevida, sin embargo, desconocemos los valores de seguridad, pudiendo estar entre 15 y $20 \mathrm{cmH}_{2} \mathrm{O}[20],[21]$. Así, al iniciar nuestra ventilación el primer parámetro es el volumen corriente, que habitualmente programamos entre seis a ocho $\mathrm{ml} / \mathrm{kg}$ IBW (Figura 3).

La programación del PEEP se establece según el potencial de reclutamiento del tejido pulmonar, el cual es muy heterogéneo de un paciente a otro[22],[23]. Existe diversas maneras de evaluar la reclutabilidad, siendo lo más usado la mecánica pulmonar[24] y la gasometría arterial, en particular la respuesta del $\mathrm{CO}_{2}$ al mantener un volumen minuto constante[22]. También, la ecocardiografía puede ser muy útil para detectar un aumento en la presión de arteria pulmonar, secundaria a las sobredistensión alveolar, y el desarrollo de un cor pulmonale agudo[25]. 

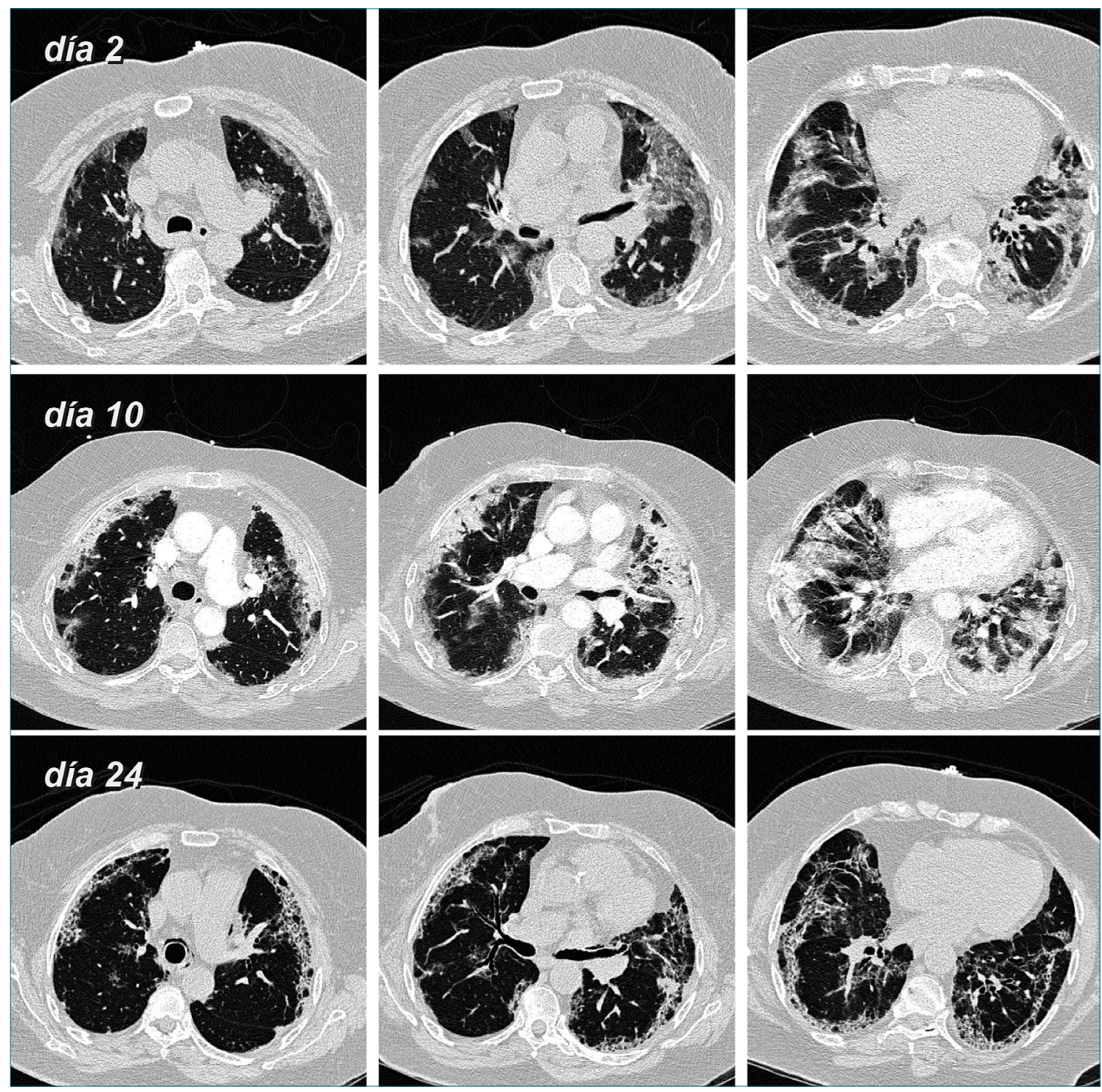

Figura 1. Tomografía computarizada en mujer de 76 años con PCR (+) para SARS-CoV-2, y que ingresa por insuficiencia respiratoria, recibiendo dexametasona (protocolo Recovery), y siendo apoyada con CNAF por varias semanas. Al segundo día de su ingreso se evidencia extensas opacidades parenquimatosas de relleno alveolar bilaterales y de predominio periférico. A la semana siguiente (día 10) se aprecia mayor compromiso y engrosamiento inter e intralobulillar, y posteriormente (día 24) hay áreas de reticulación irregular subpleural y bronquiectasias por tracción, siempre con la misma distribución anatómica.

En general, en pacientes con hipoxemia grave utilizamos altos niveles de PEEP, 10 a $15 \mathrm{~cm}$ de $\mathrm{H}_{2} \mathrm{O}$, asociado a posición prono. En fases más avanzadas de la enfermedad, en que hay un aumento de la elastancia, los niveles requeridos de PEEP tienden a ser más bajos, incluso a ZEEP (PEEP $\left.0 \mathrm{cmH}_{2} \mathrm{O}\right)$.

No existen datos con respecto a la frecuencia respiratoria, pero existe consenso en ajustar esta variable para lograr niveles de $\mathrm{PaCO}_{2}$ entre 35 y 60 , y pH entre 7,25 y 7,45, respectivamente[26]. En pacientes con hipercapnia severa, la reducción del espacio muerto instrumental es fundamental para permitir una ventilación protectora[27].

\section{Concepto de hipoxemia refractaria}

En los estadios de mayor compromiso del intercambio ga- seoso, clásicamente ante $\mathrm{Pa}: \mathrm{FiO}_{2}<150 \mathrm{mmHg}$, el uso de bloqueo neuromuscular (BNM) en infusión y el posicionamiento en prono ha mostrado ser seguros y efectivos, logrando una disminución ostensible en la mortalidad de estos pacientes[8],[28] (Figura 3). La posición prono homogeniza la distribución de la presión transpulmonar, y mejora la relación V/Q al favorecer la ventilación de los segmentos dorsales, siendo ampliamente usada y con buenos resultados durante esta pandemia[29]-[32] Así como el BNM, hemos adquirido evidencia empírica de su seguridad durante períodos prolongados, si bien requiere un adecuado entrenamiento del personal.

Las dudas quedan con respecto al momento de su suspensión, ya que muchos pacientes presentan una evolución prolongada de varias semanas. Sin haber datos al respecto, tratamos 


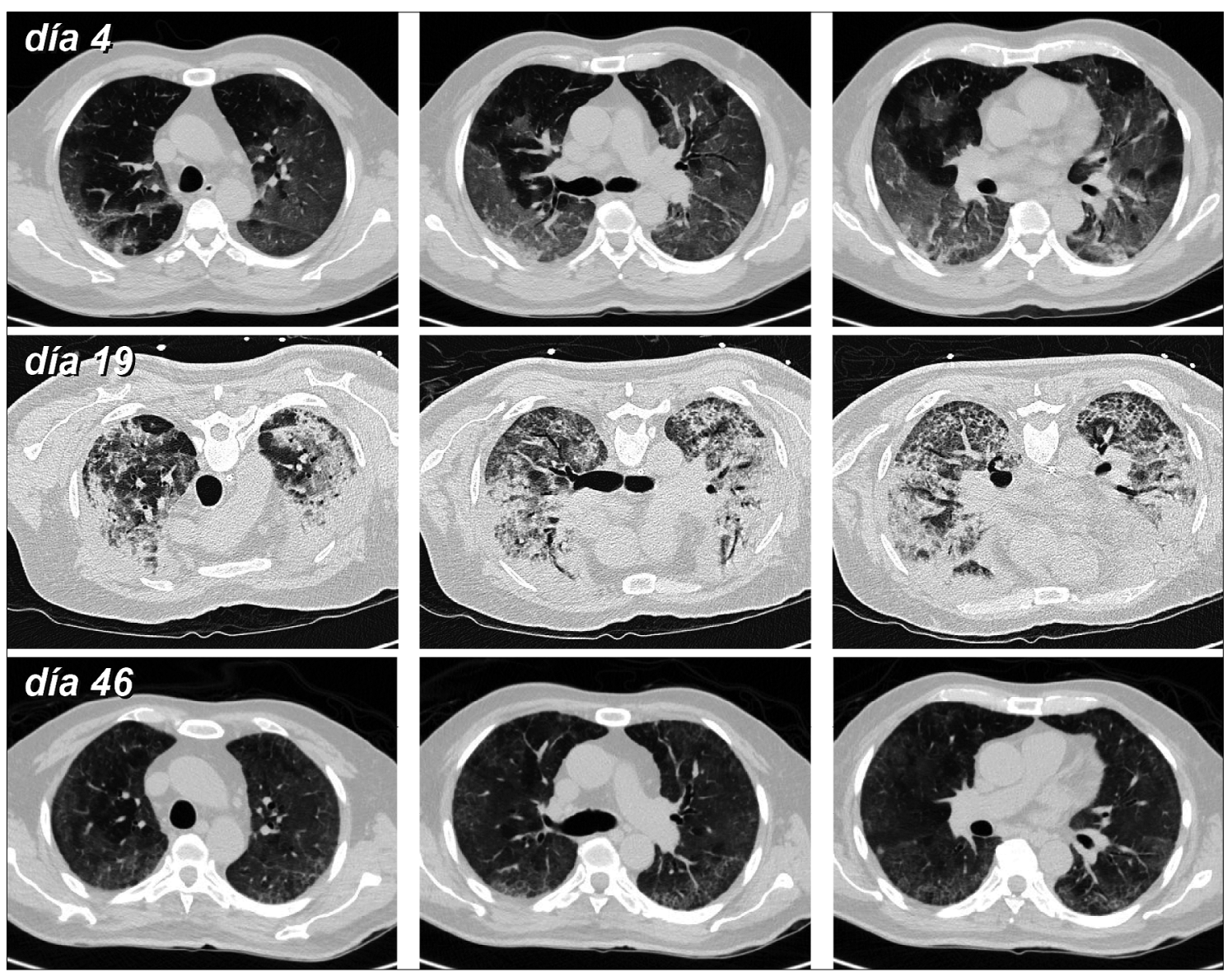

Figura 2. Tomografía computarizada en varón de 62 que ingresó por una insuficiencia respiratoria secundaria a COVID-19, siendo manejado en prono vigil y CNAF. Al $4^{\circ}$ día de ingreso se aprecia extensas opacidades multifocales con densidad en "vidrio esmerilado" de predominio en la periferia pulmonar. Cayó en ventilador al $11^{\circ}$ día de su ingreso y la TC al día 19 (en posición prono), muestra múltiples opacidades parenquimatosas condensantes periféricas, asociado a bronquiectasiasbronquielectasias por tracción y reticulaciones subpleurales (crazy paving), determinadas por neumonía en organización. El paciente fue tratado con bolos de metilprednisolona $(250 \mathrm{mg} / \mathrm{día}$ por 3 días), teniendo una positiva evolución posterior. La TC al $46^{\circ}$ día muestra gran disminución de las opacidades parenquimatosas, persistiendo tenues áreas de "vidrio esmerilado" de distribución periférica y reticulaciones periféricas y algunas bronquiectasias por tracción.

de evitar la pronación y el bloqueo neuromuscular después de la tercera semana, y sólo lo hacemos en casos seleccionados con Pa: $\mathrm{FiO}_{2}<100$.

Las maniobras de reclutamiento obedecen a la finalidad de "abrir el pulmón y mantenerlo abierto", reclutando áreas colapsadas que requieren de altas presiones de vía aérea para su ventilación y luego definiendo el mejor PEEP mediante una titulación decremental que busca la mejor distensibilidad pulmonar. Si bien la evidencia respalda su uso para evitar la hipoxemia refractaria[24], su implementación universal no ha logrado mejorías en sobrevida y su uso no se encuentra libre de riesgos[33].

\section{Oxigenación por membrana extracorpórea}

La oxigenación por membrana extracorpórea (ECMO) forma parte del último eslabón en el manejo de la hipoxemia refractaria (Figura 3). Creemos que la ventana de oportunidad para la conexión a ECMO veno-venoso está cuando el paciente cursa con hipoxemia refractaria (a pesar de posición prono, BNM y maniobras de reclutamiento), asociado a una ventilación mecánica injuriosa. Así, el ECMO cumple dos objetivos: mejorar por un lado el intercambio gaseoso, y por el otro lado bajar la

\footnotetext{
Tabla 1. Criterios para intubación orotraqueal en COVID-19 Criterios justificados

- Uso persistente de musculatura accesoria

- Respiración paradojal persistente

- Agitación o compromiso de conciencia

- Inestabilidad hemodinámica asociada a dificultad respiratoria

Criterios NO justificados

- Por alto requerimiento de $\mathrm{FiO}_{2}$ o relación $\mathrm{Pa}: \mathrm{FiO}_{2}$ baja

- Por polipnea 25-40/min

- Para permitir traslado "seguro"

- Por imagen pulmonar que muestre neumonía extensa
} 
intensidad del soporte ventilatorio, permitiendo el reposo y la recuperación pulmonar[34].

Durante la pandemia del COVID-19 se plantearon dudas respecto a la eficacia y eficiencia de esta terapia y su coste sobre los ya sobrecargados sistemas de salud. Si bien las series iniciales mostraron malos resultados clínicos, cohortes siguientes tuvieron mejores resultados[35]. En nuestro país, el ECMO ha tenido un papel importante en el rescate y traslado de pacientes con hipoxemia grave desde centros con menor experiencia[36]. La organización del sistema de salud en Chile, en conjunto con la Sociedad Chilena de Medicina Intensiva fue fundamental para la implementación de estas medidas. Sin embargo, su rol definitivo aún es discutible dada su complejidad logística y técnica, incluyendo la canulación de grandes vasos y anticoagulación. En esta línea, existen situaciones clínicas donde la conexión no está indicada o incluso contraindicada (Tabla 2)[34].

Por último, un estudio realizado en Reino Unido reportó una funcionalidad a 6 meses similar a los pacientes que no reci-

\section{Tabla 2. Contraindicaciones relativas y absolutas de ECMO}

Imposibilidad de anticoagular

Accesos vasculares limitados

Infección severa asociada

Futilidad pronosticada de terapia

Disfunción neurológica

Edad > 60 años

Inmunosupresión

Ventilación Mecánica > 7 días

bieron ECMO, pudiendo regresar a trabajar y con función pulmonar adecuada. Pese a lo anterior, los desafíos continúan en el área psicológica, donde se reportó elevadas tasas de estrés postraumático, ansiedad y depresión[37].

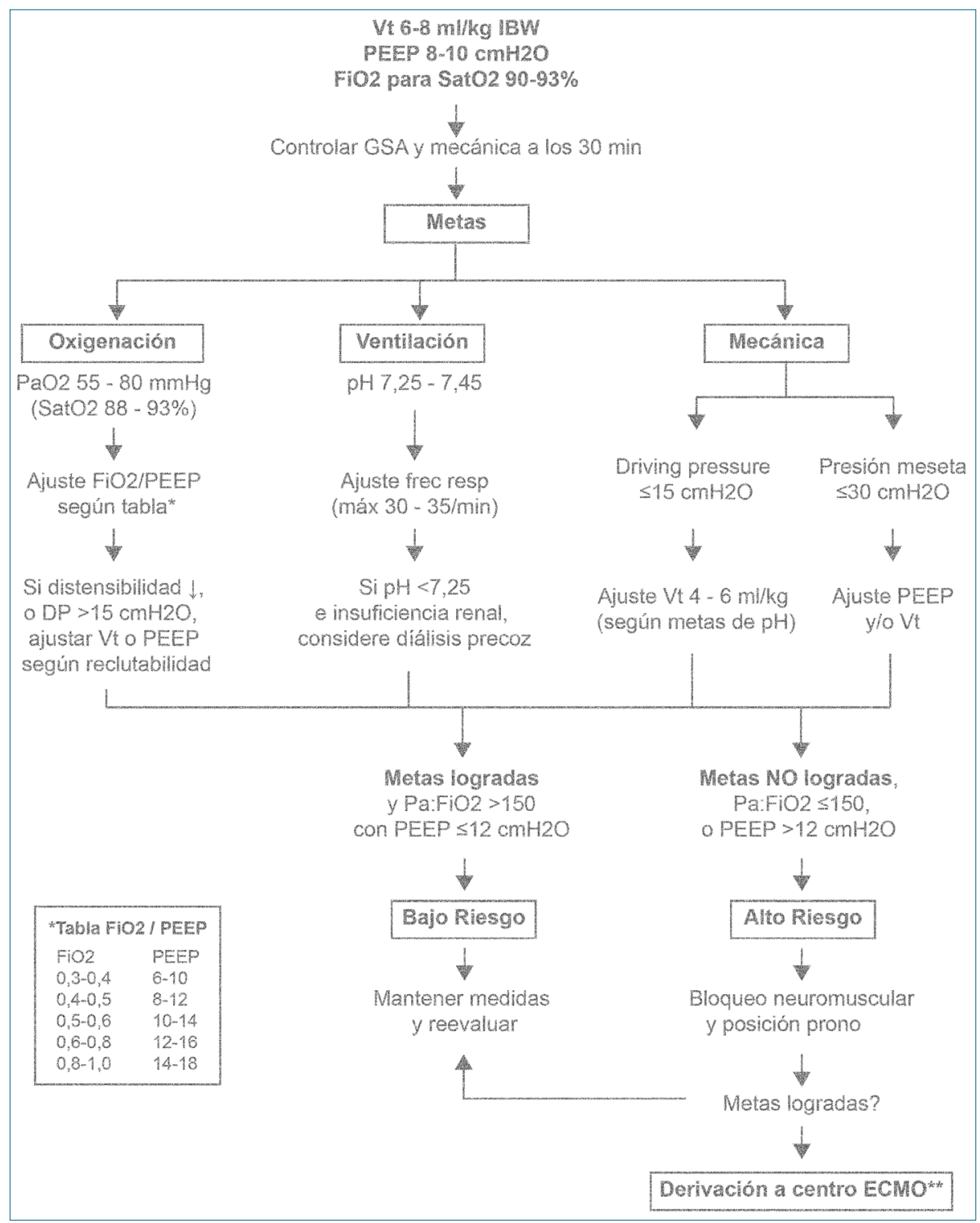

Figura 3. Protocolo de ventilación mecánica según fisiología. El manejo ventilatorio busca tres metas: oxigenación, ventilación $\left(\mathrm{PaCO}_{2}\right.$ y $\mathrm{pH}$ ) y mecánica, este último para proteger el parénquima pulmonar. Si el paciente cumple estas tres metas, tiene altas posibilidades de recuperación (bajo riesgo). Es su defecto (alto riesgo), el bloqueo neuromuscular y la posición prono están plenamente indicada. Abreviaciones: Vt: volumen corriente; $\mathrm{FiO}_{2}$ : fracción inspirada de oxígeno; DP: driving pressure (presión meseta - PEEP). 


\section{Terapias farmacológicas}

\section{Esteroides}

El gran estudio adaptativo británico RECOVERY mostró que el uso de dexametasona $6 \mathrm{mg}$ al día por 10 días disminuye la mortalidad a 28 días, la duración de las hospitalizaciones y la progresión a ventilación mecánica. Un metaanálisis posterior confirmó estos hallazgos[38]. Sin embargo, es preciso destacar que el beneficio solo se observó en aquellos que recibieron soporte con oxigenoterapia o ventilación mecánica, y no así los que no necesitaron estas medidas.

Algo característico del COVID-19 es la aparición de neumonía organizada secundaria (OP) como una complicación tardía de la enfermedad. Los hallazgos tomográficos que muestran reticulación y distorsión parenquimatosa (Figura 2), junto con biopsias y autopsias son compatibles con enfermedades pulmonares inflamatorias que responden a corticosteroides, como lo son la OP o su variante histológica, la neumonía fibrinosa aguda (AFOP)[39]. Estos hallazgos ya habían sido descritos con SARS, MERS y H1N1, en donde la OP y la AFOP se notificaron entre el $30 \%$ y $60 \%$ de los pacientes internados en la unidad de cuidados intensivos y son complicaciones bien conocidas de las infecciones virales.

Si bien la terapia RECOVERY es hoy un estándar, no tiene en consideración la aparición de la OP secundaria y su presencia muchas veces significa retrocesos en el manejo. Los esteroides son la primera línea de tratamiento para estas complicaciones, y su uso muchas veces se relaciona con mejoría en el curso clínico. De hecho, los esteroides han mostrado ser útiles en la insuficiencia respiratoria aguda hipoxémica por diversas causas[40]. Sin embargo, no hay consenso respecto al manejo en la fase tardía en COVID-19. En nuestro centro, si después de la tercera semana existe evidencia clínica y radiológica de una OP y descartada razonablemente una infección activa, iniciamos 'pulsos' de metilprednisolona, 1-2 mg/kg por día por 3 a 5 días.

\section{Anticoagulación}

Otro elemento característico del COVID-19 es la alta prevalencia de eventos trombóticos pulmonares, y que son parte de la fisiopatología de la insuficiencia respiratoria. Un metaanálisis con 66 estudios y 28.173 pacientes mostró una prevalencia de trombosis de $14,1 \%$, que aumentó a $22,7 \%$ en los pacientes ingresados a UCI[41]. Así mismo, una cohorte prospectiva observó una alta prevalencia de trombosis, en su mayoría pulmonares e incluso estando con anticoagulación profiláctica o terapéutica[42]

El estudio INSPIRATION comparó dosis intermedias de heparina de bajo peso molecular (HBPM) vs dosis estándar en 562 pacientes internados en $\mathrm{UCl}$, no encontrando diferencias, salvo más eventos hemorrágicos en el grupo de intervención[43]. Dos estudios recientes en pacientes con COVID-19 grave y moderado mostraron que el uso de dosis terapéuticas de heparina o HBPM no disminuyó los días libres de ventilación mecánica, y con más eventos hemorrágicos graves[43],[44]. Curiosamente, los pacientes con enfermedad moderada tuvieron mejores resultados en sobrevida y necesidad de soporte, aunque repitiéndose la mayor frecuencia de eventos hemorrágicos.

La evidencia actualmente disponible no favorece el uso de anticoagulación terapéutica. Sin embargo, en ausencia de contraindicaciones, se debe considerar la tromboprofilaxis en todos los pacientes hospitalizados con COVID-19[45].

\section{Otras terapias farmacológicas}

El carácter inflamatorio del COVID-19 hizo desarrollar toda una línea de investigación terapéutica para bloquear o enlentecer el avance de esta enfermedad. La interleukina-6 (IL-6) es clave en la respuesta inflamatoria y sus niveles se relacionan con la severidad de la enfermedad[46]. Sin embargo, los niveles de IL-6 son significativamente menores a los vistos en otros cuadros sépticos, lo que plantea dudas en el rol que podría tener su modulación[47].

Tocilizumab es un anticuerpo monoclonal humanizado contra el receptor de IL-6, que ha sido ampliamente utilizado en esta pandemia, pero con resultados contradictorios. Stone et al investigó si una intervención temprana con tocilizumab podría limitar la progresión a insuficiencia respiratoria o muerte, reducir el riesgo de empeoramiento clínico o disminuir el uso de oxígeno suplementario en pacientes hospitalizados por COVID-19, con resultados negativos[48]. Más tarde, el estudio REMA-CAP lograría encontrar beneficios de sobrevida en pacientes críticos con COVID-19 que reciben soporte orgánico en UCI[49]. La rama tocilizumab del estudio RECOVERY seguiría en esa línea, levantando controversia y por lo tanto, más dudas[50]. A mediados de este año, se publicó un metaanálisis con 9 estudios clínicos y 6.493 pacientes, sin encontrar una mejoría en la mortalidad[51].

Finalmente, otras terapias como la hidroxicloroquina, la ivermectina y el plasma hiperinmune, no lograron demostrar beneficio en sus respectivos ensayos clínicos y actualmente están fuera de la terapia estándar del COVID-19.

\section{El despertar y el paciente crónico}

\section{Delirium}

El delirium es un fenómeno que ocurre frecuentemente en los pacientes críticos, en particular en aquellos bajo sedación profunda y ventilación mecánica[52],[53]. Dos estudios recientes muestra una incidencia muy elevada de delirium hiperactivo (52\% y $69 \%$ ) en pacientes con COVID-19, lo que implica agitación y asincronía con el ventilador, mayor sedación, y dificultad en el destete[54]-[56]. Asimismo, al comparar cohortes históricas de pacientes no COVID-19 vs aquellos con COVID-19, los pacientes infectados por SARS-CoV-2 presentan mayores requerimientos de hipnóticos, lo que se asocia con mayor porcentaje de tiempo en coma farmacológico, y mayor mortalidad[57]. A la inversa, la mejoría en el nivel de conciencia al disminuir la sedación es un parámetro mayor que nos orienta a un paciente que va mejorando.

Múltiples factores podrían explicar la mayor incidencia de disfunción cerebral en pacientes COVID-19, incluyendo aspectos organizacionales como la restricción de visitas familiares, limitación de intervenciones no farmacológicas (p.ej. terapia ocupacional), la mayor sobrecarga laboral de los equipos de cuidados intensivos, entre otros[58]. El uso de bundles para optimizar el manejo multimodal del delirium podría impactar positivamente en los resultados clínicos, sin embargo, la literatura actual es ambivalente respecto a su real impacto. 


\section{Infecciones asociadas}

En comparación con otros cuadros sépticos, los pacientes críticos COVID-19 presentan una alta tasa sobreinfecciones del tracto respiratorio inferior traqueobronquitis y neumonía asociada a ventilador (NAV)[57]. Diversos estudios muestran tasas de NAV superior al 50\%, mucho mayor a las vistas previamente durante las epidemias invernales por influenza H1N1[58],[59].

Los mecanismos involucrados no están claros, pudiendo existir una depresión inmune secundaria a la infección misma. Sin embargo, el uso de esteroides en esta enfermedad y otros depresores del sistema inmune (tocilizumab) pueden explicar esta alta tasa de infecciones secundarias, incluido aspergillosis[59]. Además, la alta tasa de delirium y asincronía lleva muchas veces a sedar en forma excesiva, generando un círculo vicioso de mayor sedación, más infección y más delirium.

\section{El paciente crítico crónico y su rehabilitación}

La neumonía COVID-19 grave tiene un curso prolongado. Varios reportes y nuestra experiencia muestran que el tiempo promedio en el ventilador es superior a las dos semanas[29],[32],[60]. Vale decir, son pacientes críticos crónicos, con debilidad muscular, problemas nutricionales y cognitivos, e inmunodeficientes, expuestos a complicaciones infecciosas, trombóticas y hemorrágicas.

Así, después de la tercera semana evitamos pronar nuestros pacientes y suspendemos las infusiones de sedantes, que han demostrado prolongar la estadía en el ventilador[52],[61],[62]. Al promover la asistencia ventilatoria parcial, es frecuente observar frecuencias respiratorias y volúmenes minuto elevados, y driving pressures superiores a $15020 \mathrm{cmH}_{2} \mathrm{O}$ (aumento de elastancia). Nuestra conducta es tolerar volúmenes minuto hasta $250 \mathrm{ml} / \mathrm{kg} / \mathrm{min}$, y en caso de una de driving pressure aumentada, limitamos la presión meseta a $30 \mathrm{cmH}_{2} \mathrm{O}$ (Figura 3).

Si el paciente tiene expectativas razonables de recuperación, una traqueostomía puede facilitar su destete al disminuir el espacio muerto y los requerimientos de sedación y ventilación[63],[64]. Múltiples series han demostrado que la técnica percutánea (con o sin apoyo broncoscópico) es segura y eficiente en este contexto[65]. Si bien la discusión en la literatura ha estado enfocada en identificar los beneficios de la traqueostomía precoz versus tardía en el contexto COVID-19[66]-[68], creemos que hay que replantear el foco de la pregunta. Más allá de determinar la mejor temporalidad del procedimiento, hay que intentar identificar aquellos pacientes en que el procedimiento maximizará sus chances de liberarse del ventilador mecánico y de sobrevida. De este modo, evitamos traqueostomizar a pacientes que hubiesen podido destetarse exitosamente (procedimiento innecesario), y por el otro lado, someter a un procedimiento invasivo a aquellos que presentan altas chances de fallecer (procedimiento fútil)[69].

\section{Conclusiones}

Al terminar el año 2020, la vacuna contra el COVID-19 Ilegó a nuestro país con buenas referencias en términos de eficacia y seguridad. Parece ser la luz al final del túnel. Sin embargo, es muy probable que continuemos con brotes intermitentes de la enfermedad, en que debamos aplicar lo mejor de nuestros conocimientos y competencias para obtener buenos resultados clínicos.

Mientras tanto, continuemos estimulando a nuestros conocidos, parientes y amigos a mantener las medidas preventivas: uso de mascarillas, lavado frecuente de manos y distanciamiento social.

\section{Referencias}

1. Wiersinga WJ, Rhodes A, Cheng AC, Peacock SJ, Prescott HC: Pathophysiology, Transmission, Diagnosis, and Treatment of Coronavirus Disease 2019 (COVID-19): A Review. JAMA 2020, 324(8):782-793. https://doi.org/10.1001/jama.2020.12839

2. Berlin DA, Gulick RM, Martinez FJ: Severe Covid-19. N Engl J Med 2020, 383(25):2451-2460. https://doi.org/10.1056/NEJMcp2009575

3. Ackermann M, Verleden SE, Kuehnel M, Haverich A, Welte T, Laenger F, Vanstapel A, Werlein C, Stark H, Tzankov A et al: Pulmonary Vascular Endothelialitis, Thrombosis, and Angiogenesis in Covid-19. N Engl J Med 2020, 383(2):120-128. https://doi. org/10.1056/NEJMoa2015432

4. Osuchowski MF, Winkler MS, Skirecki T, Cajander S, Shankar-Hari M, Lachmann G, Monneret G, Venet F, Bauer M, Brunkhorst FM et al: The COVID-19 puzzle: deciphering pathophysiology and phenotypes of a new disease entity. Lancet Respir Med 2021, 9(6):622-642. https://doi.org/10.1016/S2213-2600(21)00218-6

5. Ehrmann S, Li J, Ibarra-Estrada M, Perez Y, Pavlov I, McNicholas B, Roca O, Mirza S, Vines D, Garcia-Salcido R et al: Awake prone positioning for COVID-19 acute hypoxaemic respiratory failure: a randomised, controlled, multinational, open-label meta-trial. Lancet Respir Med 2021.

6. Bugedo G, Pavez N, Tobar E, Bruhn A, Regueira T: Revisión de la oxigenoterapia en posición prono vigil y ventilación espontánea en pacientes con Covid-19. Beneficios y Riesgos. Revista Chilena de Medicina Intensiva 2020, 35(2).

7. Frat JP, Thille AW, Mercat A, Girault C, Ragot S, Perbet S, Prat G, Boulain T, Morawiec E, Cottereau A et al: High-flow oxygen through nasal cannula in acute hypoxemic respiratory failure. $\mathrm{N}$ Engl J Med 2015, 372(23):2185-2196. https://doi.org/10.1056/ NEJMoa1503326

8. Guerin C, Reignier J, Richard JC, Beuret P, Gacouin A, Boulain T, Mercier E, Badet M, Mercat A, Baudin O et al: Prone Positioning in Severe Acute Respiratory Distress Syndrome. The New England journal of medicine 2013. https://doi.org/10.1056/NEJMoa1214103

9. Brochard L, Slutsky A, Pesenti A: Mechanical Ventilation to Minimize Progression of Lung Injury in Acute Respiratory Failure. Am J Respir Crit Care Med 2017, 195(4):438-442. https://doi. org/10.1164/rccm.201605-1081CP

10. Carteaux G, Millan-Guilarte T, De Prost N, Razazi K, Abid S, Thille AW, Schortgen F, Brochard L, Brun-Buisson C, Mekontso Dessap A: Failure of Noninvasive Ventilation for De Novo Acute Hypoxemic Respiratory Failure: Role of Tidal Volume. Crit Care Med 2016, 44(2):282-290. https://doi.org/10.1097/ CCM.0000000000001379

11. Tonelli R, Fantini R, Tabbi L, Castaniere I, Pisani L, Pellegrino MR, Della Casa G, D'Amico R, Girardis M, Nava S et al: Early Inspiratory Effort Assessment by Esophageal Manometry Predicts Noninvasive Ventilation Outcome in De Novo Respiratory Failure. A Pilot 
Study. Am J Respir Crit Care Med 2020, 202(4):558-567. https:// doi.org/10.1164/rccm.201912-25120C

12. Grieco DL, Menga LS, Raggi V, Bongiovanni F, Anzellotti GM, Tanzarella ES, Bocci MG, Mercurio G, Dell'Anna AM, Eleuteri D et al: Physiological Comparison of High-Flow Nasal Cannula and Helmet Noninvasive Ventilation in Acute Hypoxemic Respiratory Failure. Am J Respir Crit Care Med 2020, 201(3):303-312. https:// doi.org/10.1164/rccm.201904-08410C

13. Grieco DL, Menga LS, Cesarano M, Rosa T, Spadaro S, Bitondo MM, Montomoli J, Falo G, Tonetti T, Cutuli SL et al: Effect of Helmet Noninvasive Ventilation vs High-Flow Nasal Oxygen on Days Free of Respiratory Support in Patients With COVID-19 and Moderate to Severe Hypoxemic Respiratory Failure: The HENIVOT Randomized Clinical Trial. JAMA 2021, 325(17):17311743.

14. Marini JJ, Gattinoni L: Management of COVID-19 Respiratory Distress. JAMA 2020, 323(22):2329-2330. https://doi.org/10.1001/ jama. 2020.6825

15. Tobin MJ, Laghi F, Jubran A: Caution about early intubation and mechanical ventilation in COVID-19. Ann Intensive Care 2020, 10(1):78. https://doi.org/10.1186/s13613-020-00692-6

16. Yoshida T, Amato MBP, Kavanagh BP, Fujino Y: Impact of spontaneous breathing during mechanical ventilation in acute respiratory distress syndrome. Curr Opin Crit Care 2019, 25(2):192-198. https://doi.org/10.1097/MCC.0000000000000597

17. Slutsky AS, Ranieri VM: Ventilator-induced lung injury. $N$ Engl J Med 2013, 369(22):2126-2136. https://doi.org/10.1056/NEJMra1208707

18. Ventilation with lower tidal volumes as compared with traditional tidal volumes for acute lung injury and the acute respiratory distress syndrome. The Acute Respiratory Distress Syndrome Network. N Engl J Med 2000, 342(18):1301-1308. https://doi. org/10.1056/NEJM200005043421801

19. Bruhn A, Bugedo D, Riquelme F, Varas J, Retamal J, Besa C, Cabrera C, Bugedo G: Tidal volume is a major determinant of cyclic recruitment-derecruitment in acute respiratory distress syndrome. Minerva Anestesiol 2011, 77(4):418-426.

20. Bugedo G, Retamal J, Bruhn A: Driving pressure: a marker of severity, a safety limit, or a goal for mechanical ventilation? Crit Care 2017, 21(1):199. https://doi.org/10.1186/s13054-017$1779-x$

21. Amato MB, Meade MO, Slutsky AS, Brochard L, Costa EL, Schoenfeld DA, Stewart TE, Briel M, Talmor D, Mercat A et al: Driving pressure and survival in the acute respiratory distress syndrome. N Engl J Med 2015, 372(8):747-755. https://doi.org/10.1056/ NEJMsa1410639

22. Gattinoni L, Caironi P, Cressoni M, Chiumello D, Ranieri VM, Quintel M, Russo S, Patroniti N, Cornejo R, Bugedo G: Lung recruitment in patients with the acute respiratory distress syndrome. The New England journal of medicine 2006, 354(17):17751786. https://doi.org/10.1056/NEJMoa052052

23. Bugedo G, Retamal J, Bruhn A: Does the use of high PEEP levels prevent ventilator-induced lung injury? Rev Bras Ter Intensiva 2017, 29(2):231-237. https://doi.org/10.5935/0103$507 X .20170032$

24. Kacmarek RM, Villar J, Sulemanji D, Montiel R, Ferrando C, Blanco J, Koh Y, Soler JA, Martinez D, Hernandez M et al: Open Lung Approach for the Acute Respiratory Distress Syndrome: A Pilot, Randomized Controlled Trial. Crit Care Med 2016, 44(1):32-42. https://doi.org/10.1097/CCM.0000000000001383
25. Boissier F, Katsahian S, Razazi K, Thille AW, Roche-Campo F, Leon R, Vivier E, Brochard L, Vieillard-Baron A, Brun-Buisson C et al: Prevalence and prognosis of cor pulmonale during protective ventilation for acute respiratory distress syndrome. Intensive Care Med 2013, 39(10):1725-1733. https://doi.org/10.1007/s00134013-2941-9

26. Retamal J, Damiani LF, Basoalto R, Benites MH, Bruhn A, Larsson A, Bugedo G: Physiological and inflammatory consequences of high and low respiratory rate in acute respiratory distress syndrome. Acta Anaesthesiol Scand 2021, 65(8):1013-1022. https://doi. org/10.1111/aas.13830

27. Retamal J, Libuy J, Jimenez M, Delgado M, Besa C, Bugedo G, Bruhn A: Preliminary study of ventilation with $4 \mathrm{ml} / \mathrm{kg}$ tidal volume in acute respiratory distress syndrome: feasibility and effects on cyclic recruitment - derecruitment and hyperinflation. Crit Care 2013, 17(1):R16. https://doi.org/10.1186/cc12487

28. Papazian L, Forel JM, Gacouin A, Penot-Ragon C, Perrin G, Loundou A, Jaber S, Arnal JM, Perez D, Seghboyan JM et al: Neuromuscular blockers in early acute respiratory distress syndrome. The New England journal of medicine 2010, 363(12):1107-1116. https://doi.org/10.1056/NEJMoa1005372

29. Vera M, Kattan E, Born P, Rivas E, Amthauer M, Nesvadba A, Lara $B$, Rao I, Espindola E, Rojas $L$ et al: Intubation timing as determinant of outcome in patients with acute respiratory distress syndrome by SARS-CoV-2 infection. J Crit Care 2021, 65:164-169. https://doi.org/10.1016/j.jcrc.2021.06.008

30. Mathews KS, Soh H, Shaefi S, Wang W, Bose S, Coca S, Gupta S, Hayek SS, Srivastava A, Brenner SK et al: Prone Positioning and Survival in Mechanically Ventilated Patients With Coronavirus Disease 2019-Related Respiratory Failure. Crit Care Med 2021, 49(7):1026-1037. https://doi.org/10.1097/ CCM.0000000000004938

31. Langer T, Brioni M, Guzzardella A, Carlesso E, Cabrini L, Castelli G, Dalla Corte F, De Robertis E, Favarato M, Forastieri A et al: Prone position in intubated, mechanically ventilated patients with COVID-19: a multi-centric study of more than 1000 patients. Crit Care 2021, 25(1):128. https://doi.org/10.1186/s13054-02103552-2

32. Botta M, Tsonas AM, Pillay J, Boers LS, Algera AG, Bos LDJ, Dongelmans DA, Hollmann MW, Horn J, Vlaar APJ et al: Ventilation management and clinical outcomes in invasively ventilated patients with COVID-19 (PRoVENT-COVID): a national, multicentre, observational cohort study. Lancet Respir Med 2021, 9(2):139148.

33. Writing Group for the Alveolar Recruitment for Acute Respiratory Distress Syndrome Trial I, Cavalcanti AB, Suzumura EA, Laranjeira LN, Paisani DM, Damiani LP, Guimaraes HP, Romano ER, Regenga MM, Taniguchi LNT et al: Effect of Lung Recruitment and Titrated Positive End-Expiratory Pressure (PEEP) vs Low PEEP on Mortality in Patients With Acute Respiratory Distress Syndrome: A Randomized Clinical Trial. JAMA 2017, 318(14):1335-1345.

34. Brodie D, Bacchetta M: Extracorporeal membrane oxygenation for ARDS in adults. N Engl J Med 2011, 365(20):1905-1914. https://doi.org/10.1056/NEJMct1 103720

35. Yang $X, Y u Y, X u$ J, Shu $H$, Xia J, Liu H, Wu Y, Zhang L, Yu Z, Fang $M$ et al: Clinical course and outcomes of critically ill patients with SARS-CoV-2 pneumonia in Wuhan, China: a single-centered, retrospective, observational study. Lancet Respir Med 2020, 8(5):475-481. https://doi.org/10.1016/S2213-2600(20)30079-5

36. Díaz RA, Graf J, Zambrano JM, Ruiz C, Espinoza JA, Bravo SI, 
Salazar PA, Bahamondes JC, Castillo LB, Gajardo AlJ et al: Extracorporeal Membrane Oxygenation for COVID-19-associated Severe Acute Respiratory Distress Syndrome in Chile: A Nationwide Incidence and Cohort Study. Am J Respir Crit Care Med 2021, 204(1):34-43. https://doi.org/10.1164/rccm.202011-41660C

37. Kanji HD, Chouldechova A, Harris-Fox S, Ronco JJ, O'Dea E, Harvey C, Shuster C, Thiara S, Peek GJ: Quality of life and functional status of patients treated with venovenous extracorporeal membrane oxygenation at 6 months. J Crit Care 2021, 66:26-30. https://doi.org/10.1016/j.jcrc.2021.07.010

38. Group WHOREAfC-TW, Sterne JAC, Murthy S, Diaz JV, Slutsky AS, Villar J, Angus DC, Annane D, Azevedo LCP, Berwanger $O$ et al: Association Between Administration of Systemic Corticosteroids and Mortality Among Critically III Patients With COVID-19: A Meta-analysis. JAMA 2020, 324(13):1330-1341. https://doi. org/10.1001/jama.2020.17023

39. Kory P, Kanne JP: SARS-CoV-2 organising pneumonia: 'Has there been a widespread failure to identify and treat this prevalent condition in COVID-19?'. BMJ Open Respir Res 2020, 7(1). https:// doi.org/10.1136/bmjresp-2020-000724

40. Villar J, Ferrando C, Martinez D, Ambros A, Munoz T, Soler JA, Aguilar G, Alba F, Gonzalez-Higueras E, Conesa LA et al: Dexamethasone treatment for the acute respiratory distress syndrome: a multicentre, randomised controlled trial. Lancet Respir Med 2020, 8(3):267-276. https://doi.org/10.1016/S22132600(19)30417-5

41. Zhou F, Yu T, Du R, Fan G, Liu Y, Liu Z, Xiang J, Wang Y, Song B, Gu $X$ et al: Clinical course and risk factors for mortality of adult inpatients with COVID-19 in Wuhan, China: a retrospective cohort study. The Lancet 2020, 395(10229):1054-1062. https://doi. org/10.1016/S0140-6736(20)30566-3

42. Helms J, Tacquard C, Severac F, Leonard-Lorant I, Ohana M, Delabranche X, Merdji H, Clere-Jehl R, Schenck M, Fagot Gandet F et al: High risk of thrombosis in patients with severe SARS-CoV-2 infection: a multicenter prospective cohort study. Intensive Care Med 2020, 46(6):1089-1098. https://doi.org/10.1007/s00134020-06062-x

43. Investigators $A$, Investigators $A C-a$, Investigators $R-C$, Lawler PR, Goligher EC, Berger JS, Neal MD, McVerry BJ, Nicolau JC, Gong MN et al: Therapeutic Anticoagulation with Heparin in NoncriticaIly III Patients with Covid-19. N Engl J Med 2021, 385(9):790-802. https://doi.org/10.1056/NEJMoa2105911

44. Investigators I, Sadeghipour P, Talasaz AH, Rashidi F, SharifKashani B, Beigmohammadi MT, Farrokhpour M, Sezavar SH, Payandemehr P, Dabbagh A et al: Effect of Intermediate-Dose vs Standard-Dose Prophylactic Anticoagulation on Thrombotic Events, Extracorporeal Membrane Oxygenation Treatment, or Mortality Among Patients With COVID-19 Admitted to the Intensive Care Unit: The INSPIRATION Randomized Clinical Trial. JAMA 2021, 325(16):1620-1630.

45. Piazza G, Morrow DA: Diagnosis, Management, and Pathophysiology of Arterial and Venous Thrombosis in COVID-19. JAMA 2020, 324(24):2548-2549. https://doi.org/10.1001/ jama.2020.23422

46. Leisman DE, Ronner L, Pinotti R, Taylor MD, Sinha P, Calfee CS, Hirayama AV, Mastroiani F, Turtle CJ, Harhay MO et al: Cytokine elevation in severe and critical COVID-19: a rapid systematic review, meta-analysis, and comparison with other inflammatory syndromes. Lancet Respir Med 2020, 8(12):1233-1244. https:// doi.org/10.1016/\$2213-2600(20)30404-5
47. Kox M, Waalders NJB, Kooistra EJ, Gerretsen J, Pickkers P: Cytokine Levels in Critically III Patients With COVID-19 and Other Conditions. Jama 2020, 324(15):1565-1567. https://doi.org/10.1001/ jama.2020.17052

48. Stone JH, Frigault MJ, Serling-Boyd NJ, Fernandes AD, Harvey L, Foulkes AS, Horick NK, Healy BC, Shah R, Bensaci AM et al: Efficacy of Tocilizumab in Patients Hospitalized with Covid-19. N Engl J Med 2020, 383(24):2333-2344. https://doi.org/10.1056/ NEJMoa2028836

49. Gordon AC, Mouncey PR, Al-Beidh F, Rowan KM, Nichol AD, Arabi YM, Annane D, Beane A, van Bentum-Puijk W, Berry LR et al: Interleukin-6 Receptor Antagonists in Critically III Patients with Covid-19. N Engl J Med 2021, 384(16):1491-1502. https://doi. org/10.1056/NEJMoa2100433

50. Tocilizumab in patients admitted to hospital with COVID-19 (RECOVERY): a randomised, controlled, open-label, platform trial. Lancet 2021, 397(10285):1637-1645.

51. Snow TAC, Saleem N, Ambler G, Nastouli E, Singer M, Arulkumaran N: Tocilizumab in COVID-19: a meta-analysis, trial sequential analysis, and meta-regression of randomized-controlled trials. Intensive Care Med 2021, 47(6):641-652. https://doi.org/10.1007/ s00134-021-06416-z

52. Shehabi Y, Bellomo R, Reade MC, Bailey M, Bass F, Howe B, McArthur C, Seppelt IM, Webb S, Weisbrodt L et al: Early intensive care sedation predicts long-term mortality in ventilated criticaIly ill patients. Am J Respir Crit Care Med 2012, 186(8):724-731. https://doi.org/10.1164/rccm.201203-05220C

53. Stollings JL, Kotfis K, Chanques G, Pun BT, Pandharipande PP, Ely EW: Delirium in critical illness: clinical manifestations, outcomes, and management. Intensive Care Med 2021. https://doi. org/10.1007/s00134-021-06503-1

54. Helms J, Kremer S, Merdji H, Schenck M, Severac F, Clere-Jehl R, Studer A, Radosavljevic M, Kummerlen C, Monnier A et al: Delirium and encephalopathy in severe COVID-19: a cohort analysis of ICU patients. Crit Care 2020, 24(1):491. https://doi. org/10.1186/s13054-020-03200-1

55. Pun BT, Badenes R, Heras La Calle G, Orun OM, Chen W, Raman R, Simpson BK, Wilson-Linville S, Hinojal Olmedillo B, Vallejo de la Cueva A et al: Prevalence and risk factors for delirium in critically ill patients with COVID-19 (COVID-D): a multicentre cohort study. Lancet Respir Med 2021, 9(3):239-250.

56. Damiani LF, Bruhn A, Retamal J, Bugedo G: Patient-ventilator dyssynchronies: Are they all the same? A clinical classification to guide actions. J Crit Care 2020, 60:50-57. https://doi.org/10.1016/j. jcrc.2020.07.016

57. Wongtangman $K$, Santer $P$, Wachtendorf $L J$, Azimaraghi $O$, Baedorf Kassis E, Teja B, Murugappan KR, Siddiqui S, Eikermann M, Group SOMT: Association of Sedation, Coma, and In-Hospital Mortality in Mechanically Ventilated Patients With Coronavirus Disease 2019-Related Acute Respiratory Distress Syndrome: A Retrospective Cohort Study. Crit Care Med 2021, 49(9):1524-1534. https://doi.org/10.1097/CCM.0000000000005053

58. Kotfis K, Williams Roberson S, Wilson JE, Dabrowski W, Pun BT, Ely EW: COVID-19: ICU delirium management during SARS-CoV-2 pandemic. Crit Care 2020, 24(1):176. https://doi. org/10.1186/s13054-020-02882-x

59. Povoa P, Martin-Loeches I, Nseir S: Secondary pneumonias in critically ill patients with COVID-19: risk factors and outcomes. Curr Opin Crit Care 2021, 27(5):468-473. https://doi.org/10.1097/ MCC. 0000000000000860 
60. Domecq JP, Lal A, Sheldrick CR, Kumar VK, Boman K, Bolesta S, Bansal V, Harhay MO, Garcia MA, Kaufman M et al: Outcomes of Patients With Coronavirus Disease 2019 Receiving Organ Support Therapies: The International Viral Infection and Respiratory Illness Universal Study Registry. Crit Care Med 2021, 49(3):437-448. https://doi.org/10.1097/ CCM.0000000000004879

61. Kollef MH, Levy NT, Ahrens TS, Schaiff R, Prentice D, Sherman $\mathrm{G}$ : The use of continuous i.v. sedation is associated with prolongation of mechanical ventilation. Chest 1998, 114(2):541-548. https://doi.org/10.1378/chest.114.2.541

62. Kress JP, Pohlman AS, O'Connor MF, Hall JB: Daily interruption of sedative infusions in critically ill patients undergoing mechanical ventilation. N Engl J Med 2000, 342(20):1471-1477. https://doi. org/10.1056/NEJM200005183422002

63. de la Fuente R, Kattan E, Munoz-Gama J, Puente I, Navarrete M, Kychenthal C, Fuentes R, Bravo S, Galvez V, Sepulveda M: Development of a comprehensive Percutaneous Dilatational Tracheostomy process model for procedural training: A Delphi-based experts consensus. Acta Anaesthesiol Scand 2021, 65(2):244256. https://doi.org/10.1111/aas.13716

64. Abbott F, Ortega M, Bravo S, Basoalto R, Kattan E: Can we improve teaching and learning of percutaneous dilatational tracheostomy's bronchoscopic guidance? SAGE Open Med 2021, 9:20503121211002321. https://doi. org/10.1177/20503121211002321

65. Bier-Laning C, Cramer JD, Roy S, Palmieri PA, Amin A, Anon JM, Bonilla-Asalde CA, Bradley PJ, Chaturvedi P, Cognetti DM et al: Tracheostomy During the COVID-19 Pandemic: Comparison of International Perioperative Care Protocols and Practices in 26 Countries. Otolaryngol Head Neck Surg 2021, 164(6):1136-1147. https://doi.org/10.1177/0194599820961985

66. Hernández G, Ramos FJ, Anon JM, Ortiz R, Colinas L, Masclans JR, De Haro C, Ortega A, Penuelas O, Cruz-Delgado MM et al: Early Tracheostomy for Managing ICU Capacity During the COVID-19 Outbreak: A Propensity-Matched Cohort Study. Chest 2021. https://doi.org/10.1016/j.chest.2021.06.015

67. Livneh N, Mansour J, Kassif Lerner R, Feinmesser G, Alon E: Early vs. late tracheostomy in ventilated COVID-19 patients - A retrospective study. Am J Otolaryngol 2021, 42(6):103102. https://doi. org/10.1016/j.amjoto.2021.103102

68. Battaglini D, Missale F, Schiavetti I, Filauro M, lannuzzi F, Ascoli A, Bertazzoli A, Pascucci F, Grasso S, Murgolo F et al: Tracheostomy Timing and Outcome in Severe COVID-19: The WeanTrach Multicenter Study. J Clin Med 2021, 10(12). https://doi. org/10.3390/jcm10122651

69. Boles JM, Bion J, Connors A, Herridge M, Marsh B, Melot C, Pearl $\mathrm{R}$, Silverman $\mathrm{H}$, Stanchina M, Vieillard-Baron A et al: Weaning from mechanical ventilation. Eur Respir J 2007, 29(5):1033-1056. https://doi.org/10.1183/09031936.00010206 